\title{
Burden of Haemophilus influenzae type b disease in Pakistani children
}

\author{
A. K.M. Zaidi, ${ }^{1}$ H. Khan, ${ }^{1}$ A. R. Sherali, ${ }^{2}$, R. Lasi ${ }^{7}$ and the Sindh Meningitis Study Group
}

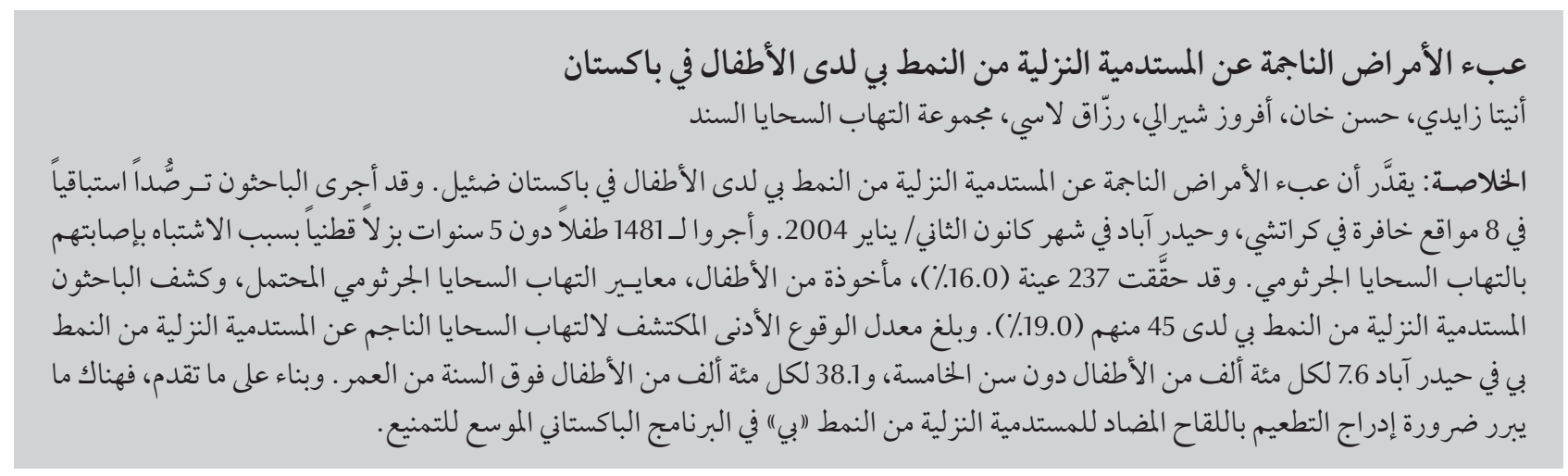

ABSTRACT Estimates of the burden of Haemophilus influenzae type b (Hib) in children in Pakistan are limited. A prospective surveillance was set up in 8 sentinel sites in Karachi and Hyderabad in January 2004. A total of 1481 children aged $<5$ years underwent lumbar puncture for suspected acute bacterial meningitis. Specimens from $237(16.0 \%)$ children met the criteria for probable bacterial meningitis, and Hib was detected in 45 of them (19.0\%). The minimum detected incidence of Hib meningitis in the Hyderabad area was 7.6 per 100000 in children $<5$ years of age, and 38.1 per 100000 children $<1$ year. Hib vaccination is justified for inclusion in Pakistan's expanded programme of immunization.

\section{Poids de l'infection à Haemophilus influenzae de type b chez les enfants pakistanais}

RÉSUMÉ L'estimation du poids de l'infection à Haemophilus influenzae de type b (Hib) chez les enfants pakistanais est limitée. Une surveillance prospective a été mise en place sur huit sites sentinelles à Karachi et Hyderabad en janvier 2004. Au total, 1481 enfants âgés de moins de cinq ans ont subi une ponction lombaire en raison d'une suspicion de méningite bactérienne aiguë. Les échantillons prélevés sur 237 enfants (16\%) présentaient les critères d'une probable méningite bactérienne, et le Hib a été détectée chez 45 d'entre eux (19\%). L'incidence de méningite à Hib la plus faible constatée dans la région d'Hyderabad était de 7,6 pour 100000 chez les enfants de moins de cinq ans, et de 38,1 pour 100000 chez ceux âgés de moins d'un an. L'inclusion du vaccin anti-Hib dans le programme élargi de vaccination du Pakistan est justifiée. 


\section{Introduction}

Haemophilus influenzae type b (Hib) is an important childhood pathogen, causing pneumonia, meningitis and sepsis, primarily in children under 5 years of age $[1-3]$. Hib has also been shown to be responsible for a high proportion of childhood pneumonia [4-6]. In industrialized countries where the Hib conjugate vaccine has been part of routine childhood immunizations for over a decade Hib invasive disease has virtually disappeared [7].

Despite the availability of the Hib vaccine for over 15 years and its proven efficacy, many obstacles have prevented developing countries in Asia and Africa from introducing it in their Expanded Programmes on Immunization (EPI). Paramount among these is that the magnitude of the disease burden and its cost to society has not been easy to demonstrate because of inadequate surveillance capacities. A review of experience in 4 developing countries showed that local surveillance data were critical in the decision to adopt Hib vaccine [8]. Another deterring factor has been the high cost of the vaccine compared to other routine vaccines in the EPI. However, many developing countries have found the vaccine to be cost-effective [9-12].

There has been much debate on the seemingly low prevalence of invasive Hib disease in Asian countries. However, Hib are fastidious organisms which are difficult to culture in resource-poor laboratories. When adequate laboratory facilities have been made available, Hib have been shown to be the leading cause of childhood meningitis in most Asian countries, including Pakistan $[13,14]$. Another important problem in estimating the burden of invasive disease due to Hib is that the most common invasive infection is pneumonia and it is very difficult to identify the etiologic agents of pneumonia in children $[15,16]$. For these reasons, the most accurate estimation of how much severe acute respiratory infection (ARI) is due to $\mathrm{Hib}$ is only available from trials of $\mathrm{Hib}$ vaccine [17].

Realizing the importance of adequate surveillance for Hib-associated disease, the World Health Organization (WHO) has developed a generic protocol for population-based surveillance [16]. This recommends that surveillance efforts in developing countries should focus on meningitis because its diagnosis is relatively straightforward and meningitis surveillance can yield very accurate estimates of Hib incidence.

The primary objective of our study was to determine the burden of meningitis due to Hib in children aged 5 years in 2 urban centres (Karachi and Hyderabad) of southern Sindh, Pakistan, by sentinel site surveillance using the WHO generic protocol. This information will enable policy-makers to make a rational decision about introducing $\mathrm{Hib}$ vaccine in Pakistan.

\section{Methods}

\section{Study setting}

In March 2004 prospective surveillance was set up in 8 sentinel sites in Karachi and Hyderabad that fulfilled the following criteria: easy accessibility for patients; more than 50 paediatric beds; 24-hour availability of skilled personnel to perform lumbar punctures for cerebrospinal fluid (CSF) analysis; and close proximity to one of the laboratory collection points of the Aga Khan University Hospital (AKUH) laboratory (none of the public sector hospitals had adequate laboratory facilities to detect Hib meningitis). These included 5 public sector hospitals (4 in Karachi, 1 in Hyderabad), 2 large private sector hospitals with high quality microbiology laboratories (both in Karachi) and 1 private university hospital (in Hyderabad).

All paediatric consultants who were members of the Sindh chapter of the Pakistan Paediatric Association were informed about the study and the availability of free CSF analysis at AKUH for any patient with suspected bacterial meningitis.

\section{Sample}

During the study period any child aged $<5$ years was eligible for the study if he/ she had undergone a lumbar puncture for clinical suspicion of acute bacterial meningitis at any of the hospitals in the surveillance system, or whose specimen had been sent to the AKUH laboratory by a paediatric consultant, and was resident in Karachi or Hyderabad.

\section{Transport of specimens and laboratory procedures}

Participating hospitals and paediatricians were requested to send CSF specimens obtained from any child clinically suspected to have acute bacterialmeningitis to their respective $\mathrm{AKUH}$ laboratory collection point. Specimens were transported to the laboratory at room temperature and underwent immediate analysis for cell count. If the cell count was $\geq 30$ cells per $\mathrm{mm}^{3}$, then latex antigen testing for Hib, pneumococci and Neisseria meningitidis was performed using Wellcogen bacterial antigen detection kits. Due to limited funding for the study and low yields from cultures due to transport delays, CSF culture was only performed on specimens with abnormal cell counts or negative latex antigen tests.

Patients with proven Hib meningitis diagnosed at 1 public sector hospital in Karachi (National Institute of Child Health) were followed for 3 months to assess complications.

\section{Reporting, data recording and analysis}

The results were reported immediately to the child's physician, as is the current practice at the AKUH laboratory. Electronic and hard copy laboratory reports were maintained by a designated laboratory technician at the AKUH laboratory. Surveillance data were entered into 
a computer in a timely fashion by one of the research officers functioning as project coordinator. Patient confidentiality was maintained and the research review and ethical review committee of AKUH approved the study.

\section{Data analysis}

Data were double-entered and analysed in SPSS, version 14.0 and Microsoft Excel.Descriptive statistics were performed for mean scores and percentages.

\section{Results}

Over the 12-month surveillance period (1 March 2004 to 28 February 2005), the surveillance system detected 1481 children aged $<5$ years who had undergone lumbar puncture for suspected acute bacterial meningitis. Over $90 \%$ of them presented at public sector hospitals. Of these 1481, specimens from 237 (16.0\%) children met the criteria for possible bacterial meningitis (cell count $>100$ per $\mathrm{mm}^{3}$, with polymorphonuclear predominance, glucose $<40 \mathrm{mg}$ / $\mathrm{dL})$. Hib wes detected in the CSF of 45 children (19.0\%) and pneumococci in 34 (14.3\%).

Of the 45 children with Hib meningitis, 28 were from Karachi and 17 from Hyderabad. Over $90 \%$ of the cases of Hib meningitis were in children < 1 year of age (20 aged $<6$ months, 22 aged $7-11$ months). All the cases from Hyderabad were $<1$ year of age. The youngest child with Hib meningitis was 11 days old. Prior use of antimicrobials before the lumbar puncture was performed was reported by 54\% of families.

Table 1 shows the surveillance results from each city for children aged $<1$ year and $<5$ years. In metropolitan Hyderabad and surrounding population (catchment approximately 222855 children $<5$ years extrapolating from the census of 1998) the minimum detected incidence of Hib meningitis in children aged $<5$ years was 7.6 per $100000(95 \%$ CI: 4.29-12.59). In Karachi, the minimum detected rate was 1.2 per 100000 children aged $<5$ years.

Follow-up information was available 3 months after discharge for a total of 15 children diagnosed with Hib meningitis at the National Institute of Child Health during the surveillance period (Table 2). Five of the 15 suffered mild to moderate neurologic sequelae and 3 had severe sequelae. There were no deaths, although the parents of 1 child who was critically ill left the hospital against medical advice and the child probably died at home.

\section{Discussion}

Hib was detected in $19.0 \%$ of children with possible bacterial meningitis in Karachi and Hyderabad, making it the commonest cause of acute bacterial meningitis in children in these cities in
Pakistan. The limitations of applying the WHO generic protocol for populationbased surveillance of Hib [16] in our setting were the requirement that catchment areas be clearly defined and health care utilization rates should be high to accurately capture all cases of meningitis - a situation that does not exist in any area of Pakistan where it would also be possible to do good surveillance and have the laboratory capacity to diagnose Hib. Therefore, the estimated rate of Hib meningitis of 38.1 per 100000 infants and 7.6 per 100000 population $<5$ years for Hyderabad is a minimum, and is likely to be a significant underestimate because we were unable (due to budgetary limitations) to capture cases from one of the public sector hospitals in the area, many smaller private hospitals were not included and many infants may have died at home without ever being admitted to a hospital.

In Karachi, the detected rate of Hib meningitis of 1.2 per 100000 in children aged $<5$ years is just the tip of the iceberg of infection because the public sector hospitals participating in the sentinel surveillance system see a very small fraction of the disease burden in Karachi, whereas in Hyderabad there are fewer private facilities that can provide care to very sick children. The 1994 National Health Survey of Pakistan found that $80 \%$ of health care activity in Pakistan occurs in the private sector [18]. In large urban cities such as Karachi, this figure is even higher. In

\begin{tabular}{|c|c|c|c|c|}
\hline \multirow[t]{2}{*}{ City/age group } & \multirow{2}{*}{$\begin{array}{l}\text { Population in this } \\
\text { age category }\end{array}$} & \multicolumn{3}{|c|}{ Hib meningitis } \\
\hline & & No. of cases & $\begin{array}{c}\text { Rate per } 100000 \\
\text { population }\end{array}$ & $95 \% \mathrm{Cl}$ \\
\hline \multicolumn{5}{|l|}{ Hyderabad } \\
\hline$<12$ months & 44571 & 17 & 38.10 & $22.35-61.40$ \\
\hline$<59$ months & 222855 & 17 & 7.60 & $4.29-12.59$ \\
\hline \multicolumn{5}{|l|}{ Karachi } \\
\hline$<12$ months & 460676 & 21 & 4.56 & $2.56-7.23$ \\
\hline$<59$ months & 2303380 & 28 & 1.21 & $0.09-1.59$ \\
\hline
\end{tabular}

${ }^{a}$ Extrapolated from 1998 census report: Pakistan census 1998, Federal Bureau of Statistics. $\mathrm{Cl}=$ confidence interval. 


\begin{tabular}{|c|c|c|}
\hline Outcome & No. of cases & $\%$ \\
\hline Normal & 6 & 40.0 \\
\hline $\begin{array}{l}\text { Mild to moderate deficit (partial hearing loss, seizure } \\
\text { disorder, mild developmental delay) }\end{array}$ & 5 & 33.3 \\
\hline $\begin{array}{l}\text { Severe sequelae (significant hearing loss or motor deficits, } \\
\text { hydrocephalus, severe developmental delay) }\end{array}$ & 3 & 20.0 \\
\hline Lost to follow-up (left hospital against medical advice) ${ }^{a}$ & 1 & 6.7 \\
\hline Deaths recorded & 0 & 0.0 \\
\hline
\end{tabular}

${ }^{a}$ Was severely ill at the time of leaving.

addition, we commonly observed that many children with suspected meningitis did not undergo lumbar puncture for a variety of reasons. Many smaller hospitals did not have skilled personnel available at night and did not have supplies for lumbar puncture, and instead empirically treated suspected meningitis with 3rd generation cephalosporins. In the larger public sector hospitals that were part of our sentinel site surveillance, many children were brought in in a very unstable condition and could not undergo lumbar puncture. There was also a fairly high refusal rate by families who consider lumbar puncture a highly invasive test, especially in private hospitals. We also observed prior antibiotic therapy reporting rates of $54 \%$ for patients undergoing lumbar puncture. Finally, our experience of working in several low-income communities of Karachi shows that health care utilization patterns are very poor, with $70 \%$ of families of sick infants refusing to take their babies to the hospital despite the provision of transport [19]. Our verbal autopsy studies in these communities show that fever with seizures is a common cause of death in infants, for which hospital care was never sought [19]. All these factors result in a serious underestimate of the burden of $\mathrm{Hib}$ meningitis. With the assumption that we may have captured only $10 \%-20 \%$ of the cases of Hib meningitis in Hyderabad we could estimate an adjusted annual incidence of 38-76 per 100000 children $<5$ years, which is substantial, and comparable to rates observed in many African and Latin American countries [16].

A notable finding is that most cases of Hib meningitis were concentrated in the very young age group, with over $90 \%$ observed in the $<1$-year-olds, and almost half in children $<6$ months of age, indicating early exposure. Hib meningitis-adjusted incidence rates in the infant age group in Hyderabad may therefore be 190-380 per 100000 children $<1$ year (using 20\% of 222855 as the denominator population of $<1$-year old-children). Therefore, the annual number of cases of Hib meningitis in children $<1$ year in Pakistan is estimated at 10 000-21 000 .

Hib meningitis represents only a fraction of the burden of invasive Hib disease, although it the most severe form of invasive disease with a high rate of permanent disability. The clinical syndrome of pneumonia is much more common, and results in many more deaths, but is under-recognized because of the difficulty of obtaining appropriate specimens. ARI is the most common cause of childhood deaths in Pakistan, responsible for $24 \%$ or 120000 deaths of the 500000 annual child deaths in Pakistan [20]. Using WHO's generic protocol for estimating Hib-related ARI childhood mortality, as well as the estimation by Black et al. [20], we derived a rate of ARI-specific mortality of 25 per 1000 children < 5 years, and Hib-specific ARI mortality of 2.3 per 1000 children $<5$ years [16]. However, whether Hib vaccine will be useful in the prevention of ARI-related mortality remains unproven in Asian countries [17].

Pakistan is a Global Alliance for Vaccines and Immunization (GAVI)eligible country, and hepatitis B virus (HBV) vaccination, in combination with diphtheria/pertussis/ tetanus (DTP), was introduced in Pakistan with GAVI support in 2006. The additional cost of introducing pentavalent vaccine including Hib conjugate (DPT-HBV-Hib) using the GAVI/ UNICEF procurement cost [21] of an additional US\$ 0.30 per dose of Hib for Pakistan works out at US\$ 5.71 million [5 500000 birth cohort $\times 3$ doses $\times 0.70$ (coverage) $\times 1.1$ (wastage $) \times$ US $\$ 1.50$ ] The cost to the government of Pakistan per Hib meningitis case averted is thus an estimated US\$268.7-537.3, making Hib vaccine a very cost-effective intervention based on preventing meningitis cases alone.

We faced many constraints in choosing appropriate surveillance sites in Karachi and Hyderabad. Public sector hospitals in the area have woefully inadequate facilities to CSF analysis, culture for Hib or perform latex antigen testing. We could not identify a single large paediatric facility in the public sector that had appropriate facilities for CSF culture or the resources to do latex antigen testing. On the other hand, these hospitals see the majority of sick children from the lower socioeconomic groups, where Hib disease would be expected to be most prevalent. Sustainable sentinel site surveillance networks for bacterial meningitis (Hib and pneumococcus) detection in public sector hospitals are needed, and a high priority should be given to improving the capacity and increasing resources for microbiology laboratories of public sector hospitals in Pakistan.

Due to budgetary constraints we could only record outcome at 3 months for children with Hib meningitis at 1 hospital in Karachi. Our limited data showed that nearly two-thirds of 
children (9 of 15) suffered significant complications with an impact on quality of life. The long-term sequelae and the resultant human and financial burden of providing care to survivors of $\mathrm{Hib}$ meningitis are also under-recognized and should be included in the estimations of vaccine cost-effectiveness.

We conclude that Hib is the commonest cause of bacterial meningitis in children in Pakistan and that there is a substantial burden of invasive Hib disease. The decision to include $\mathrm{Hib}$ in routine EPI in Pakistan from 2008 is thus warranted based on the burden of meningitis alone. Additional studies are recommended to evaluate the impact of Hib vaccine introduction on the preventable fraction of severe pneumonia.

\section{Acknowledgements}

This investigation received technical and financial support from the joint WHO Eastern Mediterranean Region (EMRO), Division of Communicable Diseases (DCD) and the WHO Special Programme for Research and Training in Tropical Diseases (TDR): the EMRO/TDRSmall Grants Scheme for Operational Research in Tropical and Other Communicable Diseases.

Members of the Sindh Meningitis Study Group (in alphabetical order): A. Ahmed (Liaquat National Hospital, Karachi), A. Bosan (National Expanded Programme of Immunization), D.S. Akram (Civil Hospital, Ka- rachi, Dow Medical College), R. Akhter (National Institute of Child Health, Karachi), M. Alavi (Aga Khan University), I. Ali (Civil Hospital, Karachi), E. Arain (Isra University, Hyderabad), S. Azeem (Aga Khan University), M. Ghani (Murshid Hospital), R. Hafiz (National Expanded Programme of Immunization), R. Hasan (Aga Khan University), Z. Isani (National Institute of Child Health, Karachi), R. Lasi (Aga Khan University), H. Memon (Civil Hospital, Hyderabad), I. Memon (Civil Hospital, Karachi), S. Qureshi (Aga Khan University), A.R. Sherali (National Institute of Child Health, Karachi, S. Shaikh (Civil Hospital, Hyderabad), R. Siyal (Civil Hospital, Hyderabad).

\section{References}

1. Funkhouser A, Steinhoff MC, Ward J. Haemophilus influenzae disease and immunization in developing countries. Reviews of infectious diseases, 1991, 13(Suppl. 6):S542-54.

2. Schillinger JA, Wenger JD, Perkins BA. Major childhood infectious diseases: bacterial meningitis. In: Murray CJL, Lopez AD, eds. The global burden of disease. Cambridge, Massachusetts, Harvard University Press, 1996.

3. Biljmer HA. World-wide epidemiology of Haemophilus influenzae meningitis; industrialized versus non-industrialized countries. Vaccine, 1991, 9(Suppl.):S5-9.

4. Lehmann D. Epidemiology of acute respiratory tract infections, especially those due to Haemophilus influenzae, in Papua New Guinea children. Journal of infectious diseases, 1992, 165:S20-5.

5. Mulholland EK, Hilton S, Adegbola R. Randomized trial of Haemophilus influenzae type-b tetanus protein conjugate vaccine for prevention of pneumonia and meningitis in Gambian infants. Lancet, 1997, 349:1191-7.

6. Levine OS, Lagos R, Munoz A. Defining the burden of pneumonia in children preventable by vaccination against Hib. Pediatric infectious disease journal, 1999, 18:1060-4.

7. Peltola H, Kilpi T, Antila M. Rapid disappearance of Haemophilus influenzae type $\mathrm{b}$ meningitis after routine childhood immunization with conjugate vaccines. Lancet, 1992, 340:592-4.

8. Wenger JD et al. Introduction of Hib conjugate vaccines in the non-industrialized world: experience in four "newly adopting countries". Vaccine, 1999, 18:736-42.

9. Hussey GD, Lasser ML, Reekie WD. The costs and benefits of a vaccination programme for Haemophilus influenzae type $b$ disease. South African medical journal, 1995, 85:20-5.

10. Limcango MR et al. Cost-benefit analysis of a Haemophilus influenzae type $\mathrm{b}$ meningitis prevention programme in The Philippines. Pharmacoeconomics, 2001, 19:391-400.
11. Levine OS et al. Cost-benefit analysis for the use of Haemophilus influenzae type b conjugate vaccine in Santiago, Chile. American journal of epidemiology, 1993, 137:1221-8.

12. Miller MA. An assessment of the value of Haemophilus influenzae type b conjugate vaccine in Asia. Pediatric infectious disease journal, 1998, 17:S152-9.

13. Peltola $\mathrm{H}$. Need for Haemophilus influenzae type $\mathrm{b}$ vaccination in Asia as evidenced by epidemiology of bacterial meningitis. Pediatric infectious disease journal, 1998, 17:S148-51.

14. Bhutta ZA. Burden of Hemophilus influenzae and Streptococcus pneumoniae infections during childhood in Pakistan. Journal of the College of Physicians and Surgeons-Pakistan, 2000, 10:346-54.

15. Isaacs D. Problems in determining the etiology of communityacquired childhood pneumonia. Pediatric infectious disease journal, 1989, 8:143-8.

16. Levine OS et al. Generic protocol for population-based surveillance of Haemophilus influenzae type $b$. Geneva, World Health Organization, 1996 (WHO/VRD/GEN/95.05).

17. Gessner BD et al. Incidences of vaccine-preventable Haemophilus influenzae type b pneumonia and meningitis in Indonesian children: hamlet-randomized vaccine-probe trial. Lancet, 2005, 365:43-52.

18. National health survey of Pakistan. Islamabad, Government of Pakistan, 1994

19. Zaidi AKM, Bhutta ZA. Care-seeking in low-income urban and semi-urban populations of Karachi. Karachi, Aga Khan University, 2007.

20. Black RE, Morris S, Bryce J. Where and why are 10 million children dying every year? Lancet, 2003, 361:2226-34.

21. Co-financing policy and country grouping document. GAVI Alliance [online document] 2007 (http://www.gavialliance.org/ resources/Co_financing_policy_and_country_grouping_document.doc, accessed 15 November 2009). 